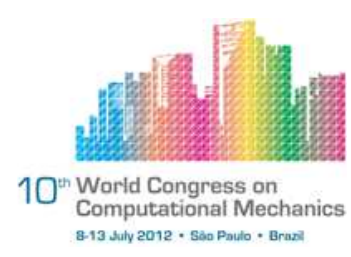

\title{
ANALYSIS OF STABILITY CONDITIONS OF A SLENDER BEAM UNDER WIND EFFECTS USING NUMERICAL MODEL
}

\author{
R. Král ${ }^{1}$, J. Náprstek ${ }^{2}$ \\ ${ }^{1}$ Department of Dynamic and Stochastic Mechanic, Institute of Theoretical and Applied Me- \\ chanics (kral@itam.cas.cz) \\ ${ }^{2}$ Department of Dynamic and Stochastic Mechanic, Institute of Theoretical and Applied Me- \\ chanics
}

\begin{abstract}
The paper deals with a numerical analysis of an aero-elastic system wherein the flow-structure interaction is taking place in its entire complexity. It is addressed to flowinduced vibrations and other response types termed as flutter or divergence, which arise due to fluctuating fluid forces and movement of the vibrating body. These phenomena are investigated on two different types of a slender beam cross-section. This strategy enables to model the body as a double-degree of freedom (DDOF) system. The paper demonstrates a determination of aero-elastic behavior of the body before and within the critical state. The attention is paid to the elastic stiffness, damping and other DDOF system parameters influence on the type and shape of aero-elastic stability limits. The question of aero-elastic stability with regard to external disturbances is discussed as well. The relevant bifurcation points and hysteresis in response loops as functions of the wind speed are identified. A separation curve emerges within the hysteresis loop dividing the space into trivial and post-critical regimes. The results obtained using numerical approach are described and widely interpreted physically. Extensive comparison with results obtained by both (i) analytical investigation and (ii) wind tunnel experiments is presented.
\end{abstract}

Keywords: Stability conditions, Aero-elastic response, Flutter.

\section{INTRODUCTION}

Long-span bridges together with light foot-bridges rank among engineering structures for which assessment of the wind load is important. When such a bridge is exposed to the wind, the static response is generated that is accompanied by time dependent component or even a regime with the self-excited force arises. Dynamic response and self-excited forces, often of stochastic character, depends on many factors which are associated with dynamic properties of a bridge and shape of a bridge girder.

For most bridge deck cross-sectional shapes, unless they are very streamlined, a bluff-body separated flow rather than potential is generated in the wake that has ever complex character. 
In the past, an examination of aero-elastic effects on bridge structures was completely a matter of wind tunnel tests, see e.g., [1,2]. This was done usually through the application of methods of system identification to the oscillatory response and force records of wind-tunnel models. Though having drawbacks resulting from the reduced scale of a model, this way of assessment still keeps the main position. Nevertheless, in the recent years the rapid onset of the computation technology clearly showed wide possibilities of the numerical simulation and many computational techniques for fluid flow simulation concerning elastic body interaction have arisen. Some papers dealing with the determination of aero-elastic characteristics of a slender beam in the wind can be found e.g., in $[3,4]$ or for a stationary body condition see e.g., $[5,6]$.

Major problems of cable-supported bridges under wind conditions are those associated with excess deflection, caused either by random response due to the action of wind gusts or by oscillatory instability. Whilst the first occurs at very high wind speeds leading to random buffeting oscillations, oscillatory instability may have its origin at much lower wind velocities. Vortex-shedding oscillations generated by alternating von Kármán vortices are ranked among the fluid-induced vibrations that generally appear at lower wind speed. At higher wind velocities, truly unstable, divergent oscillations termed as flutter can occur. These oscillations take place at a certain wind velocity as self-excited oscillations caused by aerodynamic forces, see e.g., [7]. This type of excitation can depend, but not necessarily, on so-called mode coupling. This is when motion occurs in two (or more) interactive modes of vibration. The structure susceptibility to this instability, called classical (or coupled) flutter, depends generally on ratio of natural frequencies of the two modes related to the fundamental modes of vibration, see [8]. In addition, it also depends on energy supplied to the system by wind as well as on system dissipation. Although a bridge deck may oscillate at mode-coupling vibrations, single mode phenomena, such as static divergence or vortex-shedding oscillation, can also be observed as well as buffeting.

In this paper, two different slender cross-section beams (representing bridge girders, for instance) under the wind load are investigated. The analysis supposes the full fluid-structured interaction of a double-degree of freedom system based on the Newton 's fluid, elasto-dynamic equations and transformation equations. Compatibility of computational domains with different physical character is achieved by means of Lagrange multipliers defined on the interface boundaries. Numerical instabilities within the Navier-Stokes equations associated with the high Reynolds number are reduced to acceptable level using procedure combining the Galerkin-Petrov and Least-Square variation principles. The paper demonstrates a determination of aero-elastic behavior of the body before and within the critical state. The attention is paid to the elastic stiffness, damping and other DDOF system parameters influence on the type and shape of aero-elastic stability limits. The question of aero-elastic stability with regard to external disturbances is discussed as well. Procedure of slow wind speed incrementing up to the onset of individual types of critical states and later subsequent decrementing of that is used in order to identify relevant bifurcation points and hysteresis in response loops as functions of the wind speed. A separation curve emerges within the hysteresis loop dividing the space into trivial and post-critical regimes. The results obtained using numerical approach are described and widely interpreted physically. Comparison with results obtained by both 
analytical investigation and wind tunnel experiments is presented.

\section{Numerical modelling of flexible mounted beam under the wind}

Every solid structure is generally enclosed with at least one fluid. Movement of the structure generates a motion of the fluid around with adequate force effect on the structure. With respect to the mutual cohesion governed by a few kinematic and dynamic conditions, the fluid and structure is considered as a coupled system (FSI) and multi-physics model must be employed respecting dynamic equations.

Important consideration when simulating FSI problems is the choice of an appropriate kinematic description of the flow field. Generally, a computational mesh is deformed throughout the simulation according to the displacement of the solid domain where each node of the computational mesh follows the material particle. This description of motion is called Lagrangian algorithm. Simulation of the turbulent flows requires a different approach, where the computational mesh is fixed and the fluid moves with respect to the grid. This condition may be fulfilled by applying the Eulerian algorithm. On the other hand, the key drawback of this method is the difficulty to follow free surfaces and interfaces between different material or different media. By combining the two mentioned approaches, the best advantage is achieved. The arbitrary Lagrangian-Eulerian (ALE) method, as the kinematic description is called, is based on the introduction of a computational mesh which can move with a velocity independent of the velocity of the material particles, see e.g., [9]. Nevertheless, due to the ALE method, the transformation equations must be introduced thereby making the boundary problem more complicated.

\subsection{Relations of transformation}

So as to establish the deformable domain resulting from the cross-section motion, we first introduce essential expressions involved in the transformation equations. Let $\boldsymbol{x}=\{x, y\}^{T}$ be the coordinates of deformed mesh and $\boldsymbol{X}=\{X, Y\}^{T}$ stands for the fixed mesh. The deformed coordinates depend on the fixed coordinates and on time. Then the transformation stated in the matrix format reads as:

$$
\left[\begin{array}{l}
d x \\
d y
\end{array}\right]=\left[\begin{array}{ll}
x_{X} & x_{Y} \\
y_{X} & y_{Y}
\end{array}\right]\left[\begin{array}{l}
d X \\
d Y
\end{array}\right]=J\left[\begin{array}{l}
d X \\
d Y
\end{array}\right],
$$

where the subscript in Eq. (1) denotes the derivative with respect to the fixed domain coordinate. To formulate the reverse transformation, multiply this equation by the Jacobian inverse matrix $J^{-1}$ to obtain:

$$
\left[\begin{array}{l}
d X \\
d Y
\end{array}\right]=J^{-1}\left[\begin{array}{l}
d x \\
d y
\end{array}\right]
$$

where

$$
J^{-1}=\frac{1}{D_{J}}\left[\begin{array}{cc}
y_{Y} & -x_{Y} \\
-y_{X} & x_{X}
\end{array}\right]=\left[\begin{array}{cc}
I_{X x} & I_{X y} \\
I_{Y x} & I_{Y y}
\end{array}\right] \text {, with } D_{J}=x_{X} y_{Y}-x_{Y} y_{X}
$$


By applying the transformation mapping and the inverse Jacobian matrix, we can write the derivatives using the fixed coordinate variables:

$$
\left[\begin{array}{ll}
x_{x} & y_{x} \\
x_{y} & y_{y}
\end{array}\right]=J^{-1}\left[\begin{array}{ll}
x_{X} & y_{X} \\
x_{Y} & y_{Y}
\end{array}\right]=\left[\begin{array}{l}
x_{X} I_{X x}+x_{Y} I_{Y x} y_{X} I_{X x}+y_{Y} I_{Y x} \\
x_{X} I_{X y}+x_{Y} I_{Y y} y_{X} I_{X y}+y_{Y} I_{Y y}
\end{array}\right] .
$$

These transformation expressions will be further used for substitution of spacial derivatives with respect to the deformable coordinate system that appear in the weak-form equations of both the dynamic equation of Newtonian fluid and Lagrangian-Eulerian description of motion.

\subsection{Arbitrary Lagrangian-Eulerian description of motion}

The two considered coordinate systems are connected by mapping:

$$
\left.x(X, Y, t), y(X, Y, t), X, Y \in \Omega_{f}, x, y \in \Omega_{d}, t \in\right] 0, T[
$$

where upper-case letters correspond to the fixed domain $\Omega_{f} \subset \mathbb{R}^{2}$ with smooth boundary $\partial \Omega_{f}$, lower-case letters correspond to the deformed domain $\Omega_{d} \subset \mathbb{R}^{2}$, and $t$ is time. Let us assume that the set of equations governing the transformation between Lagrangian and Eulerian approaches are defined over domain $\Omega^{m}, \Omega^{m} \subset \Omega_{d}$. The Dirichlet boundary conditions giving values to the problem on $\partial \Omega^{m}$ are referred to as $\partial \Omega_{D}^{m}$ and it consists of the exterior boundary $\partial \Omega_{e x t}^{m}$ enclosing $\Omega^{m}$ and the inner boundary (interface) $\partial \Omega_{I}$ established between the solid body and fluid. The interface is defined by the shape of the structural domain $\Omega^{s}$ (slender beam). The model solves the moving coordinate system and the moving mesh in the flow channel for each time instance by applying Laplace's equation on the velocity of the mesh $\boldsymbol{\theta}(x, y, t)=\left\{\theta_{1}, \theta_{2}\right\}^{T}$ and a partial equation governing the mesh displacement $\Psi(x, y, t)=\left\{\Psi_{1}, \Psi_{2}\right\}^{T}$ :

$$
\begin{aligned}
\nabla^{2} \boldsymbol{\theta} & =\mathbf{0} & & \text { on } \left.\Omega^{m} \forall t \in\right] 0, T[, \\
\frac{d \boldsymbol{\Psi}}{d t}-\boldsymbol{\theta} & =\mathbf{0} & & \text { on } \left.\Omega^{m} \forall t \in\right] 0, T[, \\
\boldsymbol{\Psi}(x, y, 0) & =\{X, Y\}^{T} & & \text { on } \Omega^{m}, \\
\boldsymbol{\theta} & =\mathbf{r} & & \text { on } \partial \Omega_{D}^{m},
\end{aligned}
$$

where

$$
\nabla^{2}=\sum_{i=1}^{2} \frac{\partial^{2}}{\partial x_{i}^{2}}
$$

and $\mathbf{r}$ is a vector prescribing the Dirichlet portion of the boundary $\partial \Omega_{D}^{m}$ such that:

$$
\begin{array}{ll}
\boldsymbol{r}=\mathbf{0} & \text { on } \partial \Omega_{\text {ext }}^{m}, \\
\boldsymbol{r}=\dot{\mathbf{d}} & \text { on } \partial \Omega_{I} .
\end{array}
$$

With regard to the essential requirements of compatibility conditions at the fluid-structure interface, the Lagrange multiplier technique is employed and therefore the vector of compatibility conditions based on the relaxation of the boundary condition $\boldsymbol{\theta}=\mathbf{r}$ on $\partial \Omega_{D}^{m}$ can be 
directly obtained. It is done by adding linear constraint equations to the weak form of the set of equations (5) and (6) and the weak formulation of the ALE problem takes the following mixed formulation:

Given $\boldsymbol{r}(t)$, find $\left.\boldsymbol{\theta}(x, y, t) \in \mathcal{H}^{1}\left(\Omega^{m}\right) \times\right] 0, T\left[\boldsymbol{\Psi}(x, y, t) \in \mathcal{L}_{2}\left(\Omega^{m}\right) \times\right] 0, T[$ and $\left.\boldsymbol{\lambda}_{f} \in \mathbb{M}^{\prime}\left(\partial \Omega_{D}^{m}\right) \times\right] 0, T[$ such that

$$
\begin{array}{cl}
-\mathrm{d}(\hat{\boldsymbol{\theta}}, \boldsymbol{\theta})+\left(\gamma \hat{\boldsymbol{\theta}}, \boldsymbol{\lambda}_{f}\right)_{\partial \Omega}=0 & \forall \hat{\boldsymbol{\theta}} \in \mathcal{H}^{1}\left(\Omega^{m}\right), \\
\left(\hat{\boldsymbol{\Psi}}, \Psi_{t}\right)-(\hat{\boldsymbol{\Psi}}, \boldsymbol{\theta})=0 & \forall \hat{\boldsymbol{\Psi}} \in \mathcal{L}_{2}\left(\Omega^{m}\right), \\
\left(\hat{\boldsymbol{\lambda}}_{f}, \gamma \boldsymbol{\theta}\right)_{\partial \Omega}-\left(\hat{\boldsymbol{\lambda}}_{f}, \boldsymbol{r}\right)_{\partial \Omega}=0 & \forall \hat{\boldsymbol{\lambda}}_{f} \in \mathbb{M}^{\prime}\left(\partial \Omega_{D}^{m}\right),
\end{array}
$$

where d $(\hat{\boldsymbol{\theta}}, \boldsymbol{\theta})=\int \nabla \hat{\boldsymbol{\theta}}: \nabla \boldsymbol{\theta} d \Omega^{m}$ and $\left(\gamma \hat{\boldsymbol{\theta}}, \boldsymbol{\lambda}_{f}\right)_{\partial \Omega}=\int \gamma \hat{\boldsymbol{\theta}} \boldsymbol{\lambda}_{f} d \partial \Omega^{m}$ is the scalar product in $\mathcal{L}_{2}\left(\partial \Omega^{m}\right)$. Here $\mathbb{M}$ is the space in which we seek the multipliers $\boldsymbol{\lambda}_{f}$ and it is constructed using the trace operator $\gamma: \mathcal{H}^{1}\left(\Omega^{m}\right) \rightarrow \mathcal{H}^{1 / 2}\left(\partial \Omega^{m}\right)=\mathbb{M}\left(\partial \Omega^{m}\right)$ and $\mathbb{M}^{\prime}=\mathcal{H}^{-1 / 2}\left(\partial \Omega^{m}\right)$ is the dual space to $\mathbb{M}$. Note that the classical Dirichlet boundary $\boldsymbol{\theta}=\boldsymbol{r}$ is fulfilled because it is included into the variation problem. Unknown $\boldsymbol{\lambda}_{f}$ gives the following meaning:

$$
\boldsymbol{\lambda}_{f}=\frac{\partial \boldsymbol{\theta}}{\partial \boldsymbol{n}}
$$

where $\boldsymbol{n}$ is the outward unit normal vector along $\partial \Omega^{m}$.

\subsection{Wind flow simulation}

We consider the following velocity-pressure formulation of the Navier-Stokes Equations (NSE), governing unsteady incompressible flows on the domain $\Omega^{m}$ (the domain matches the ALE domain) in the moving mesh coordinates:

$$
\begin{array}{rlrl}
\rho \frac{\partial \boldsymbol{u}}{\partial t}-\nabla\left[(-p) \mathbf{I}+\eta\left(\nabla \boldsymbol{u}+(\nabla \boldsymbol{u})^{T}\right)\right]+\rho((\boldsymbol{u}-\boldsymbol{\theta}) \cdot \nabla) \boldsymbol{u} & =\mathbf{0} & & \text { on } \left.\Omega^{m} \forall t \in\right] 0, T[ \\
-\nabla \cdot \boldsymbol{u} & =0 & & \text { on } \left.\Omega^{m} \forall t \in\right] 0, T[(10 \\
\boldsymbol{u} & =\boldsymbol{z} & & \text { on } \partial \Omega_{D}^{m}, \\
{\left[(-p) \mathbf{I}+\eta\left(\nabla \boldsymbol{u}+(\nabla \boldsymbol{u})^{T}\right)\right] \cdot \mathbf{n}=\boldsymbol{h}} & & \text { on } \partial \Omega_{N}^{m}, \\
\boldsymbol{u}(x, y, 0)-\boldsymbol{u}_{0}(x, y) & =\mathbf{0} & & \text { on } \Omega^{m},
\end{array}
$$

where $\rho$ is the density of the fluid, $\boldsymbol{u}=\left\{u_{1}, u_{2}\right\}^{T}$ is the velocity field of the flow with reference to $\{x, y\}^{T}$ Cartesian directions, $p$ is the fluid dynamic pressure, $\eta$ is dynamic viscosity and I is the unit diagonal matrix. $\boldsymbol{\theta}=\left\{\theta_{1}, \theta_{2}\right\}^{T}$ stands for the velocity field of the mesh. The equilibrium equation (9) is described by three terms. The first one ensures temporal evolution of the problem, the second term represents the Cauchy stress tensor and the last one is the nonlinear convective term pertaining to the moving mesh. The incompressibility condition is governed by Eq. (10). Similarly as in the ALE problem, the vector with prescribed values $\boldsymbol{z}$ on boundary $\partial \Omega_{D}^{m}$ consists as follows:

$$
\begin{array}{ll}
\boldsymbol{z}=\boldsymbol{u}_{D} & \text { on } \partial \Omega_{e x t}^{m}, \\
\boldsymbol{z}=\dot{\mathbf{d}} & \text { on } \partial \Omega_{I}, \\
z=p_{0} & \text { on } \partial \Omega_{e x t}^{m} .
\end{array}
$$


In practice, solutions to a convection dominated transport problem by the Galerkin method are often corrupted by spurious node-to-node oscillations. We might detect these instabilities in the studied fields, primarily where steep gradients are presented. One way to free the oscillation is to refine the mesh in a exposed place. It is clear that this solution undermines the practical utility of FE method. In our case, GLS stabilisation for incompressible flow is used where the stabilising terms added are obtained by minimising the sum of the squared residual of the momentum equation integrated over each element domain, see [10,11]. We solve the NSE in the deformed coordinates, but compute the solution in fixed coordinates. The weak-form integral for the stabilised Navier-Stokes equations may be stated as follows: given $\boldsymbol{z}(t), \mathbf{h}$, find the velocity field $\left.\boldsymbol{u}(x, y, t) \in \mathcal{H}^{1}\left(\Omega^{m}\right) \times\right] 0, T[$, the pressure field $p(x, y, t) \in$ $\left.\mathcal{H}^{1}\left(\Omega^{m}\right) \times\right] 0, T\left[\right.$ and multipliers $\left.\boldsymbol{\lambda}_{u}(t), \lambda_{p}(t) \in \mathbb{M}^{\prime}\left(\partial \Omega_{D}^{m}\right) \times\right] 0, T\left[\right.$, for all $\hat{\boldsymbol{u}}, \hat{p} \in \mathcal{H}^{1}\left(\Omega^{m}\right)$ and $\hat{\boldsymbol{\lambda}}_{u}, \hat{\lambda}_{p} \in \mathbb{M}^{\prime}\left(\partial \Omega_{D}^{m}\right)$ such that:

$$
\begin{array}{r}
\left(\hat{\boldsymbol{u}}, \rho \boldsymbol{u}_{t}\right)+\mathrm{a}(\hat{\boldsymbol{u}}, \boldsymbol{u}) \eta-\mathrm{b}(\hat{\boldsymbol{u}}, p)+\mathrm{c}(\boldsymbol{u}-\boldsymbol{\theta} ; \hat{\boldsymbol{u}}, \boldsymbol{u})+\rho\left(\gamma \hat{\boldsymbol{u}}, \boldsymbol{\lambda}_{u}\right)_{\partial \Omega_{D}^{m}}+ \\
+\sum_{k=1}^{n_{e l}} \tau_{e}\left(\left(\rho((\boldsymbol{u}-\boldsymbol{\theta}) \cdot \nabla) \hat{\boldsymbol{u}}-\eta \nabla^{2} \hat{\boldsymbol{u}}, \mathbf{R e s}\right)_{\Omega^{e}}\right)-(\hat{\boldsymbol{u}}, \boldsymbol{h})_{\partial \Omega_{N}^{m}}=0 \\
-\mathrm{b}(\hat{p}, \boldsymbol{u})+\left(\gamma \hat{p}, \lambda_{p}\right)_{\partial \Omega_{D}^{m}}-\sum_{k=1}^{n_{e l}} \tau_{e}(\nabla \hat{p}, \mathbf{R e s})_{\Omega^{e}}=0 \\
\left(\hat{\boldsymbol{\lambda}}_{u}, \gamma \boldsymbol{u}\right)_{\partial \Omega_{D}^{m}}-\left(\hat{\boldsymbol{\lambda}}_{u}, \boldsymbol{z}\right)_{\partial \Omega_{D}^{m}}=0 \\
\left(\hat{\lambda}_{p}, \gamma p\right)_{\partial \Omega_{D}^{m}}-\left(\hat{\lambda}_{p}, z\right)_{\partial \Omega_{D}^{m}}=0
\end{array}
$$

where $\mathrm{a}(\hat{\boldsymbol{u}}, \boldsymbol{u})=\int \nabla \hat{\boldsymbol{u}}: \nabla \boldsymbol{u} d \Omega^{m} ; \mathrm{b}(\hat{\boldsymbol{u}}, p)=\int \nabla \hat{\boldsymbol{u}} \cdot p d \Omega^{m}$

$$
\begin{aligned}
& \mathrm{c}(\boldsymbol{u}-\boldsymbol{\theta} ; \hat{\boldsymbol{u}}, \boldsymbol{u})=\int \hat{\boldsymbol{u}} \cdot((\boldsymbol{u}-\boldsymbol{\theta}) \cdot \nabla) \boldsymbol{u} d \Omega^{m} ; \\
& \operatorname{Res}=\left(\rho \frac{\partial \boldsymbol{u}}{\partial t}-\eta \Delta \boldsymbol{u}+\rho((\boldsymbol{u}-\boldsymbol{\theta}) \cdot \nabla) \boldsymbol{u}+\nabla p\right) .
\end{aligned}
$$

The stabilisation is achieved by adding two terms with a certain weighting factor into the Eqs (14) and (15). The added term in (14) involves the second derivatives of the weighting function $\hat{\boldsymbol{u}}$, although the solution of the Galerkin-Lagrange form of NSE admits for the trial functions $\boldsymbol{u}$ and $p$ to belong in space $\mathcal{H}^{1}$ and $\mathcal{L}_{2}$, respectively. Considering linear approximation functions, the terms with second derivatives under the summation vanish which reduces to the convective term in (14) and pressure gradient in (15). The stabilisation terms involve the element-level integrals on sub-domain $\Omega^{k}, k=1,2 \ldots, n_{e l}$, where $n_{e l}$ is the number of elements. It is obvious that stabilisation parameter $\tau$ must vanish when the mesh is refined (as stated above, no stabilisation is necessary for a fine enough mesh).

The coefficient $\tau$ is obtained by a simple multi-dimensional generalisation of the optimal $\tau$ given in $[10,12]$ for one dimensional space-time formulation using family of methods, $\phi \in$ ] 0,1 , for time discretisation. The expression for the $\tau$ is:

$$
\tau=\left(\left(\frac{1}{\phi \Delta t}\right)^{2}+\left(\frac{2\left\|\boldsymbol{u}^{h}\right\|}{h^{e}}\right)^{2}+9\left(\frac{4 \eta}{\rho\left(h^{e}\right)^{2}}\right)^{2}\right)^{-1 / 2},
$$

where $\eta$ is the dynamic viscosity, and $\Delta t$ and $h$ are time step and "element length", often taken as the diameter of the circle which is area-equivalent to the element. The first term in Eq. (18) 
is associated with the temporal evaluation. A value of the parameter $\phi$ depends on a method employed for the time discretisation. The remaining two terms correspond with the fourthorder accurate formula and share both in the additional stability for flows at a large Reynolds number and in feasibility of the use of mixed elements with equal-order interpolations for velocity and pressure.

To trace the transient response, this system of semi-discrete equations can be advanced in time by suitable finite difference schemes such as the $\theta$ family methods. Note that a fully implicit method requires the solution of a nonlinear algebraic system at each time step. Semiimplicit methods in which the convection matrix $\mathbf{C}(\boldsymbol{u}, \boldsymbol{\theta}(t))$ is treated explicitly are thus generally preferred.

All relations for the NSE here have been derived in the Eulerian form and they ignore the domain deformation with respect to the section motion. To work with it, we introduce the transformation equations established for the ALE problem in the Navier-Stokes problem.

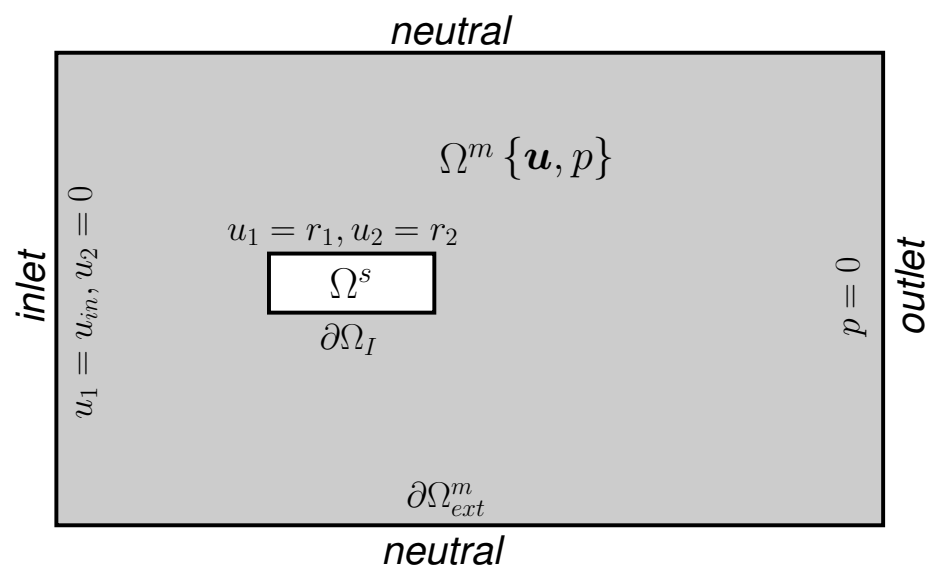

Figure 1. Fluid-structure geometry scheme with boundary conditions specified for fluid domain.

As an initial condition of the transient NSE, a linear static solution $\boldsymbol{u}_{0}(x, y)$ is applied considering the same boundary conditions as used in the transient problem. This preliminary operation serves to find an initial time step for the time-dependent solver leading, among others, towards a considerable decrease in time. In many cases, it is the only available procedure to start a simulation.

There are external and interface boundaries. The external boundaries $\partial \Omega_{\text {ext }}^{m}$ are divided into three essential parts - inlet, outlet and neutral, see Figure 1. All of these boundaries are constant in time except velocity $u_{i n}$ at inlet boundary that can change in time. The moving interface between the fluid and the structural domain is denoted $\partial \Omega_{I}$.

Dirichlet boundary conditions are prescribed at the inlet and outlet external boundaries and at the interface. On the inlet boundary the free-stream velocity $u_{i n}$ with no turbulence is assumed in $X$-direction and zero velocity in $Y$-direction. Upper and lower boundaries $\partial \Omega_{e x t}^{m}$ accomplish neutral conditions. On the outlet external boundary a "natural" boundary condition is assumed in the form of $\left[(-p) \mathbf{I}+\eta\left(\nabla \mathbf{u}+(\nabla \mathbf{u})^{T}\right)\right] \mathbf{n}=\mathbf{0}$, where $\mathbf{n}$ is the unit outward normal vector, and the Dirichlet boundary condition is the zero outlet pressure $p=0$. 
This does not correspond to real conditions of the prototype and therefore to prevail over this fact, it is proper to locate the section far enough away from the outlet boundary.

\subsection{Classical linear elastodynamics}

Let $\Omega^{s}$ be the bounded domain in $\mathbb{R}^{d}, d=2$, with smooth boundary $\partial \Omega_{I}$. The dynamic response of the structure defined over $\Omega^{s}$ can be solved by the elastodynamics equation in the form:

$$
\begin{aligned}
\rho^{s} \frac{\partial^{2} \boldsymbol{d}}{\partial t^{2}}-\nabla \boldsymbol{\sigma}^{s} & =\boldsymbol{f}(x, y, t) & & \text { on } \left.\Omega^{s} \forall t \in\right] 0, T[, \\
\boldsymbol{d} & =\boldsymbol{s} & & \text { on } \left.\partial \Omega_{D}^{s} \forall t \in\right] 0, T[, \\
\boldsymbol{d}(x, y, 0) & =\{X, Y\}^{T} & & \text { on } \Omega^{s}
\end{aligned}
$$

with the displacement $d_{i}(t)$ for $i=1,2$ defined as the difference between the current position and initial position $\{X, Y\}^{T}, \sigma_{i j}^{s}$ for $i, j=1,2$ denotes the (Cauchy) stress tensor and $f_{i}$ stands for an external force. The stress tensor $\boldsymbol{\sigma}^{s}$ is defined in terms of the strain tensor by the generalised Hook's law:

$$
\sigma_{i j}^{s}=c_{i j k l} \epsilon_{k l},
$$

where the elastic coefficients $c_{i j k l}$ are, in general, given function in a spatial dimensions $\boldsymbol{x}$. Relaxing the strong form of the equation of motion (19), the variational formulation is provided: Let $\mathbb{J}=\mathcal{H}^{1}\left(\Omega^{s}\right)$ denote the trial solution space and $\mathbb{V}=\mathcal{H}^{1}\left(\Omega^{s}\right)$ the variation space. Each member $d_{i} \in \mathbb{J}$ satisfies $\boldsymbol{d}=\boldsymbol{s}$ on $\partial \Omega_{D}^{s}$, whereas each $\hat{d}_{i} \in \mathbb{V}$ satisfies $\hat{d}_{i}=$ 0 on $\partial \Omega_{D}^{s}$. The weak formulation goes as follows:

Given $\boldsymbol{f}, \boldsymbol{d}(x, y, 0)$, find $\boldsymbol{d} \in \mathbb{\mathbb { N }}$ such that for $\hat{\boldsymbol{d}} \in \mathbb{V}$ :

$$
\left(\hat{\boldsymbol{d}}, \rho^{s} \ddot{\boldsymbol{d}}\right)+\mathrm{a}(\hat{\boldsymbol{d}}, \boldsymbol{d})=(\hat{\boldsymbol{d}}, \boldsymbol{f})
$$

where a $(\hat{\boldsymbol{d}}, \boldsymbol{d})=\int \hat{d}_{(i, j)} c_{i j k l} d_{(k, l)} d \Omega^{s}$ and $(\hat{\boldsymbol{d}}, \boldsymbol{f})=\int \hat{d}_{i} f_{i} d \Omega^{s}$. Coefficient $\rho^{s}$ stands for the material density. When combined with an appropriate boundary condition on the displacement, Eq. (22) leads to the positive-definiteness of the matrix.

For easier orientation in the dependent variables of multi-physics problem, see Figure 2.

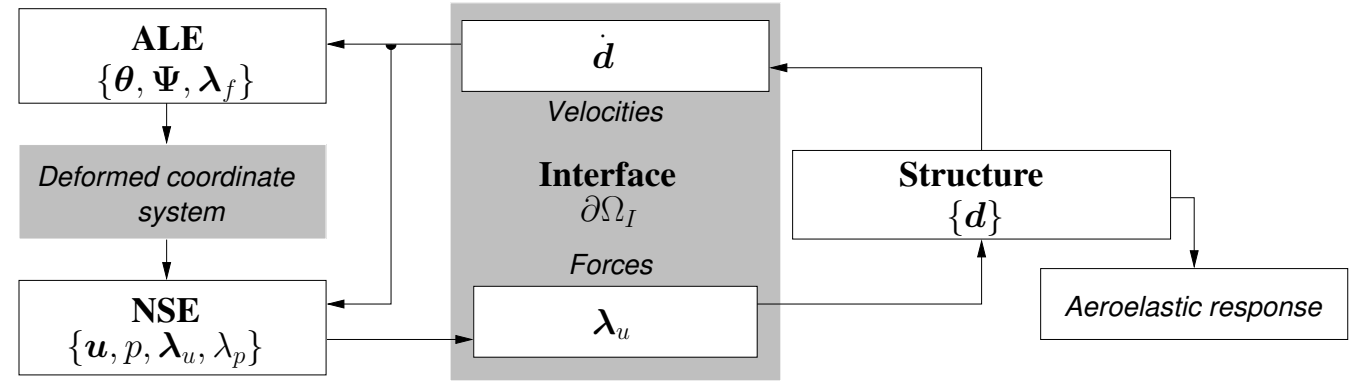

Figure 2. Multiphysics diagram of dependent variables. 


\subsection{Force vector and modal characteristics implementation}

Internal forces $\boldsymbol{f}(x, y, t)$ defined in the previous section over $\Omega^{s}$ represents volume forces given by the material characteristics of the body. Regarding the force effects on the body edges generated by the wind, a new form of the force description must be employed. Assume that the force transfer from the fluid to structural domain is carried out by means of Lagrange multiplier $\boldsymbol{\lambda}_{u}$ on each mesh node on the interface $\partial \Omega_{I}$. Furthermore, we know that the structural domain $\Omega^{s}$ is surrounded by the fluid domain $\Omega^{m}$ and thus the flexible motion has to be specified in terms of a fictitious form. Introducing nodal force vector $\mathbf{r}_{\text {nodal }}$ over $\Omega^{s}$, relation

$$
r_{\text {nodal }, i C}=r_{F, i C} \quad C \in \eta_{F} \text { for } i=1,2 \text { on } \Omega^{s} \forall t \in R^{+}
$$

shall prescribe the force in specific nodes $\eta_{F}$ on $\Omega^{s}$, and for the fluid effects holds

$$
r_{\text {nodal }, i \hat{A}}=\lambda_{u, i \hat{A}} \quad \hat{A} \in \eta_{D i} \text { for } i=1,2 \text { on } \partial \Omega_{I} \forall t \in R^{+},
$$

where $r_{F, i C}(d, \dot{d})$ means the fictitious forces ensuring the flexible mounting with reference to the considered modal properties and $r_{\text {nodal }, i \hat{A}}$ results from the solution of the NavierStokes problem.

Taking into account (23) and (24), the elastodynamic system (22) takes the following form of ordinary second order differential system:

$$
\mathbf{M} \ddot{\mathbf{d}}(t)+\mathbf{K d}(t)=\mathbf{f}(t)+\mathbf{r}_{\text {nodal }}(t),
$$

which respects both the flexible mounting of the body and wind actions.

To implement dynamic behaviour with controlled modal properties, let us assume that the section is totally rigid and the system enables movement in two generalised coordinates vertical and rotation. This idealised conception helps for understanding the virtual mounting and to determine adequate forces in terms of the dynamic properties of the model. Such a system can be appropriately described by the equation of dynamic equilibrium with 2DOFs leading to the matrix form:

$$
\mathbf{M}^{r} \ddot{\mathbf{d}}^{r}+\mathbf{f}_{F}\left(d^{r}, \dot{d}^{r}\right)=\mathbf{0} \quad \forall t \in R^{+}
$$

where $\mathbf{M}^{r}=\operatorname{diag}\left(m_{i i}^{r}\right), i=1,2$ is mass matrix with concentrated mass, $\mathbf{d}^{r}=\{h, \alpha\}^{T}$ is response vector and $\mathbf{f}_{F}=\mathbf{C}^{r} \dot{\mathbf{d}}^{r}+\mathbf{F}^{r} \mathbf{d}^{r}$, stands for the virtual forces consisting of dissipative and stiffness matrices $\mathbf{C}^{r}=\operatorname{diag}\left(c_{i i}^{r}\right)$ and $\mathbf{F}^{r}=\operatorname{diag}\left(f_{i i}^{r}\right)$, respectively. These matrices determine the eigen-frequencies and damping characteristics of the idealised dynamic system, all of the $i$-th mode.

The concept of fictitious mounting is depicted in Figure 3. It is represented on a rectangular, however, an arbitrary shape can be investigated analogously. This dummy system, replacing a standard body attachment based upon definite boundary conditions, sustains the body in the equilibrium position by means of two pairs of forces $F_{h}, F_{t}$ and $C_{h}, C_{t}$. Aforementioned coupling involves in the stiffness in heave and rotation, respectively; the later denotes damping forces. If some displacement occurs during the simulation, forces are automatically generated respecting modal properties of the dynamic system. Note that the motion in $X$-direction is totally fixed. 


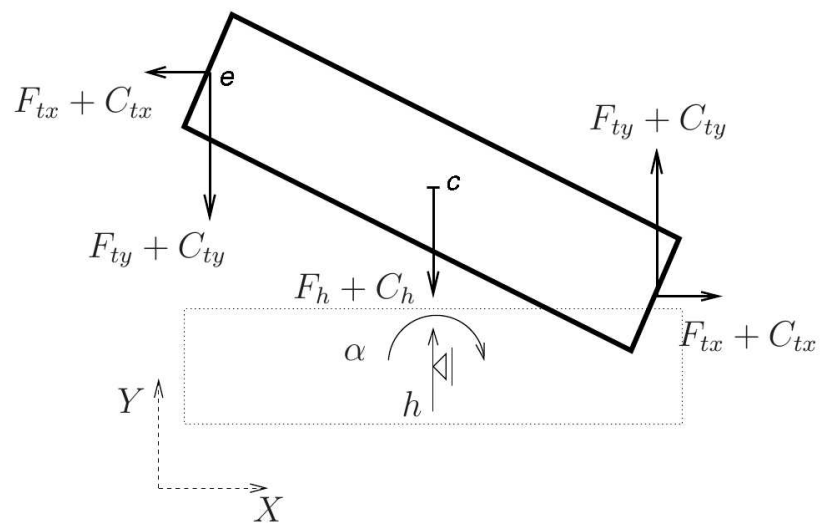

Figure 3. Force equilibrium notation determining two degrees of freedom response.

The force in the heave motion reacts in the centre of gravity of the section (symbol $c$ ), while the forces ensuring the torsion are located in the centre of leading and trailing edges (symbol $e$ ). Values of the forces and mass characteristic are derived in relation to the demanded modal characteristics according to the following equations:

$$
\begin{aligned}
& F_{t x}=-\frac{B}{2} f_{\alpha \alpha}^{r} \alpha \sin (\alpha), \quad F_{t y}=-\frac{B}{2} f_{\alpha \alpha}^{r} \alpha \cos (\alpha), \\
& C_{t x}=-\frac{B}{2} c_{\alpha \alpha}^{r} \dot{\alpha} \sin (\alpha), \quad C_{t y}=-\frac{B}{2} c_{\alpha \alpha}^{r} \dot{\alpha} \cos (\alpha),
\end{aligned}
$$

for the rotational motion, and

$$
F_{h}=-f_{h h}^{r} d_{2, c}, \quad C_{h}=-c_{h h}^{r} \dot{d}_{2, c},
$$

for the heave motion. The angle of the rotation $\alpha$ and its derivative $\dot{\alpha}$ in Eqs (27) - (28) can be formulated as:

$$
\alpha=\arcsin \left(\frac{d_{2, e}-d_{2, c}}{B / 2}\right), \quad \dot{\alpha}=\frac{1}{\sqrt{1-\left(\frac{d_{2, e}-d_{2, c}}{B / 2}\right)^{2}}}\left(\frac{\dot{d}_{2, e}-\dot{d}_{2, c}}{B / 2}\right),
$$

where $B$ denotes the section width, $d_{1, e}$ and $d_{2, c}$ are component of displacement in $X$-direction at point $e$ and in $Y$-direction at point $c$, respectively. Parameters $f_{\alpha \alpha}^{r}, f_{h h}^{r}$, that appertain to spring stiffness in matrix $\mathbf{F}^{r}$, result from the desired values of the undamped natural frequencies of the system, $\omega_{\alpha}, \omega_{h}$ :

$$
f_{\alpha \alpha}^{r}=\frac{2 \cdot \omega_{\alpha}^{2} \cdot I_{p}}{B^{2}}, \quad f_{h h}^{r}=m \cdot \omega_{h}^{2},
$$

where $I_{p}$ is polar moment of inertia and $m$ is its mass. It is obvious that specific weight $\rho$ (mass per unit volume) and hence mass, $m$, depends on the polar moment of inertia, $I_{p}$. Regarding the structural damping parameters, for $c_{\alpha \alpha}^{r}$ and $c_{h h}^{r}$, holds:

$$
c_{\alpha \alpha}^{r}=\frac{4 I_{p} \omega_{\alpha} \cdot \delta_{\alpha}}{\sqrt{\delta_{\alpha}^{2}+4 \pi^{2}} \cdot B^{2}}, \quad c_{h h}^{r}=2 m \omega_{h} \cdot \delta_{h}
$$

These last four formulae (31) - (32) define the response of the structure proportionally damped to the velocity. This viscous damping is given to both degrees of freedom by the 
logarithmic decrements $\delta_{\alpha}, \delta_{h}$. The intensities of stiffness and dissipative forces arise from an instantaneous position and velocity of the body. This means that the computational accuracy is affected by the length of the time step in a time solver. In principle, with respect to the slow response of the body examined and high oscillation period compared to the chosen time step, negligible differences in force increments can occur.

\section{Fundamental characteristics of numerical simulation}

Based on the theory defined previously, two fundamental prismatic beams are numerically examined in the smooth flow. Bluff body in the rectangular shape with aspect ratio of $1: 5$ together with streamlined section designed according to the existing bridge deck are considered, see Figure 4 left. These sections are free oscillating in the vertical bending and torsion mode with specific frequencies as depicted in Figure 4 right. A movement in the lateral direction is fixed. Each of modes is proportionally damped at various rate with respect to the body velocity. Furthermore, we assume that the beams are subjected to the wind action with a constant velocity profile and no turbulence.

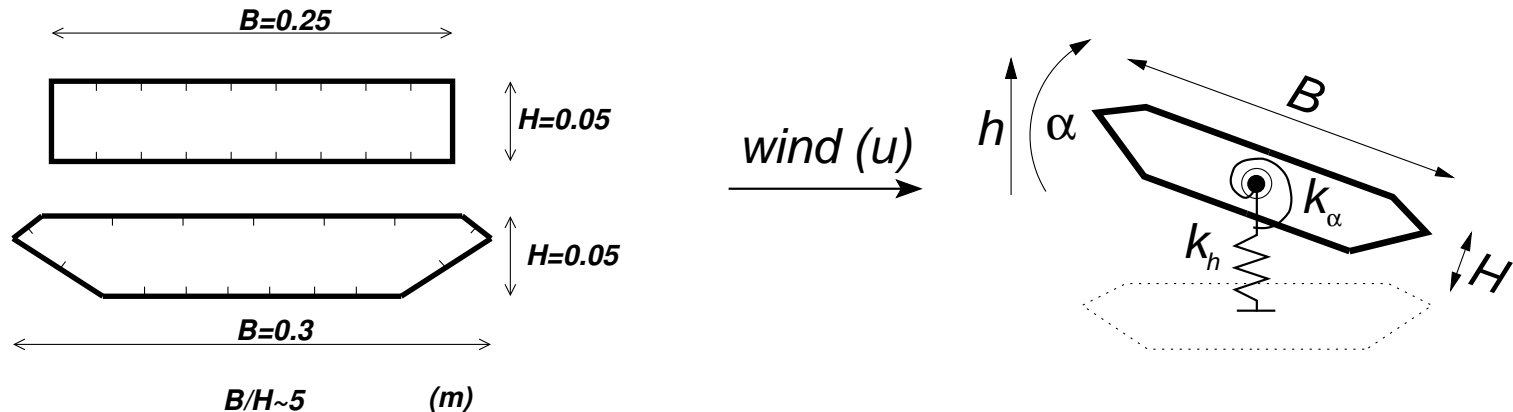

Figure 4. Profiles examined in the fluid-structure interaction (left); Beam movement with positive orientation to the wind direction.

The simulations are performed with Comsol software. This partly-open computation package solves an arbitrary weak-form of integral-differential equations with boundary conditions. During the simulations, the Reynolds number $R e=u H / \nu$ is in the range of 12300 to 92300 , provided the characteristic dimension is $H=0.05 \mathrm{~m}$. The values of $R e$ numbers are consistent with the wind-tunnel experiments. Note that dynamic viscosity and density of the air are $\eta=1.71 \cdot 10^{-5} \mathrm{kgm}^{-1} \mathrm{~s}^{-1}$ and $\rho=1.23 \mathrm{kgm}^{-3}$, respectively.

Table 1. Parameters of examined profiles.

\begin{tabular}{lcc}
\hline Profile & $\square$ & $\rightleftharpoons$ \\
\hline width, $B$ & $0.25 \mathrm{~m}$ & $0.305 \mathrm{~m}$ \\
length, $L$ & & $0.6 \mathrm{~m}$ \\
moment of inertia, $I$ & $0.0204 \mathrm{kgm}$ & $0.0225 \mathrm{kgm}$ \\
weight, $m$ & $3.76 \mathrm{~kg}$ & $3.73 \mathrm{~kg}$ \\
angular frequency in heave, $\omega_{h}$ & $\langle 25.44-32.15\rangle \mathrm{rad} \mathrm{s}^{-1}$ \\
angular frequency in rotation, $\omega_{\alpha}$ & \multicolumn{2}{c}{$30.22 \mathrm{rad} \mathrm{s}^{-1}$} \\
damping ratio in heave, $\xi_{h}$ & 0.008 & 0.0089 \\
damping ratio in rotation, $\xi_{\alpha}$ & 0.0168 & 0.0159 \\
\hline
\end{tabular}


In Table 1, fundamental parameters used in the analysis are available. In order to conform the experiments in the wind tunnel, the length of the prisms $L$ corresponds to the physical model and all characteristics related to the model length are thus adapted.

Wind effects on the bodies are investigated for various wind velocities. Each simulation is beforehand extended along the non-dimensional time interval $t_{r e d}=\frac{t u}{B} \in\langle 0,60\rangle$ to overcome a transient process between the initial condition $\boldsymbol{u}_{0}$ and the steady-state flow.
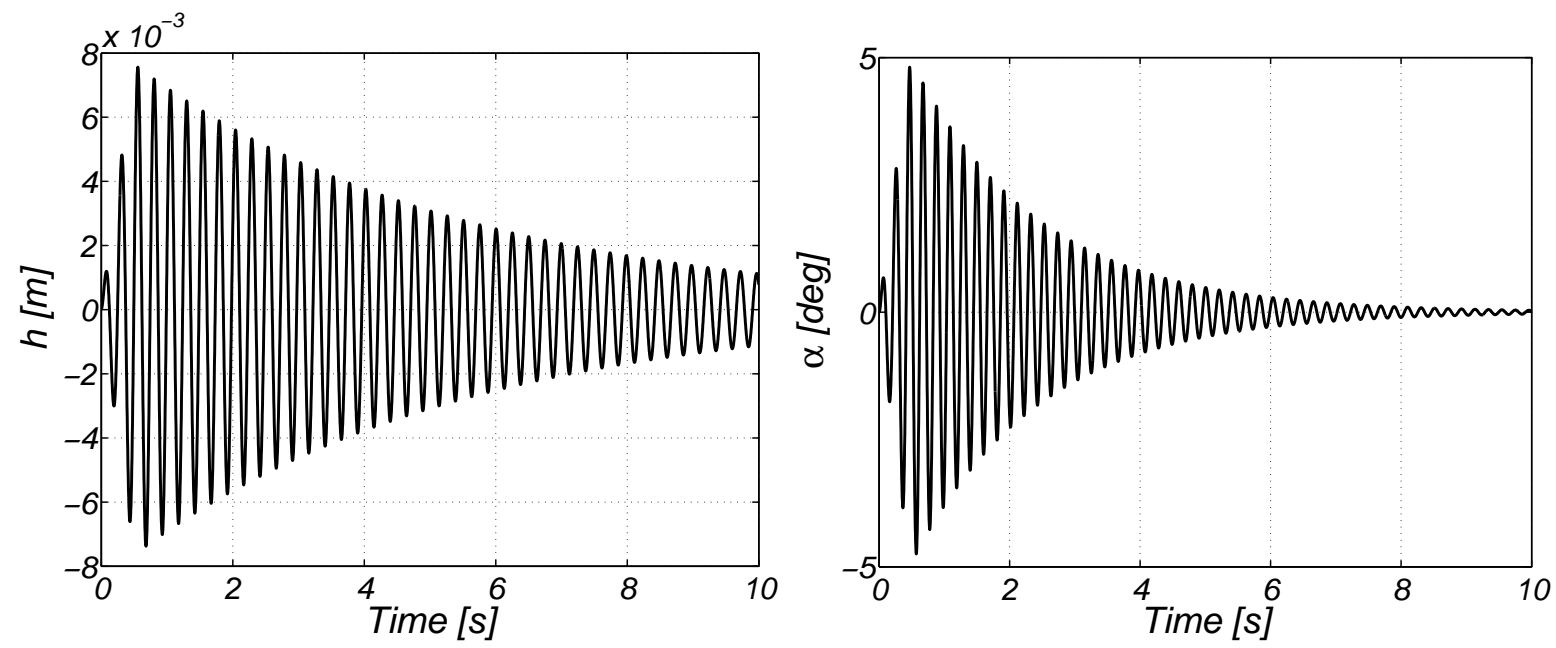

Figure 5. Trial analysis of the fictitious section mounting; Heave (left) and rotary motion (right).

To verify the fictitious attachment system, the sections were excited by a short-time force in both degrees of freedom simultaneously. This trial analysis has as one's task to prove the mutual independence of the motion and to check correct rate of the damping ratio and modal properties. Figure 5 depicts representative time-history signals of both degrees of freedom. Excepting marked fall of amplitudes, the signals do not sick for beats or any events representing the mutual interaction between DOFs.
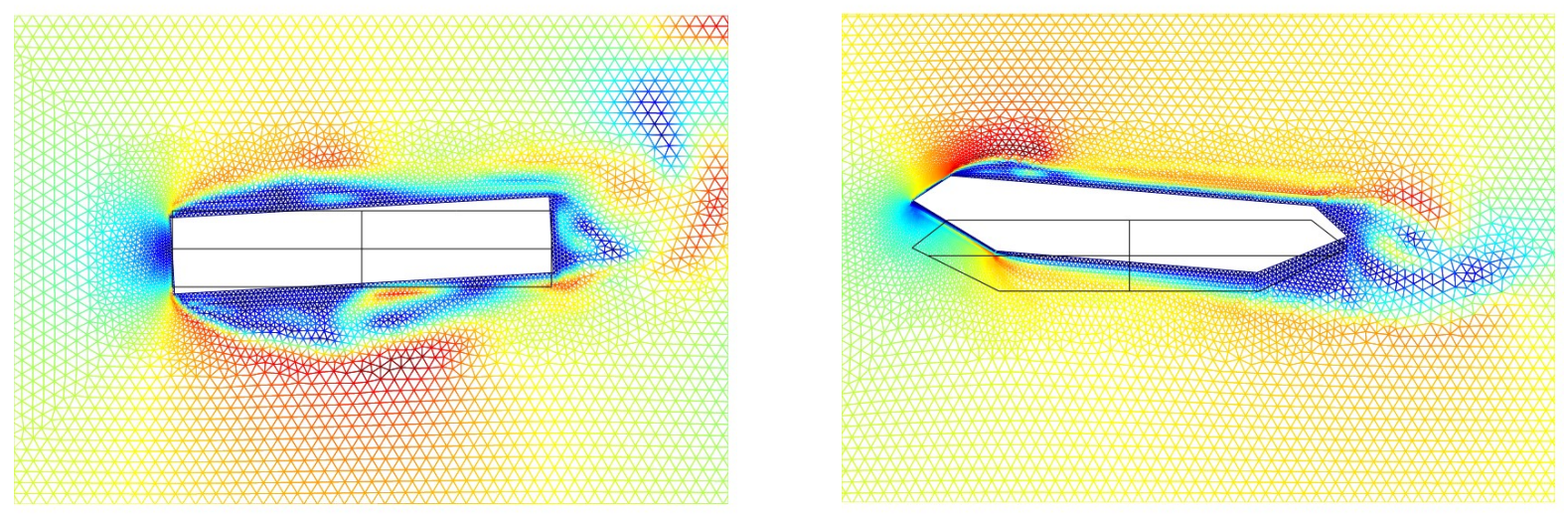

Figure 6. The finite element mesh in the deformed form for both types of cross-sections; Colouring defines instantaneous velocity field generated around.

Finite element discretisation is performed under a compromise respecting the computational accuracy and computational costs. The highest grid density is used in the vicinity of the 
section. With the distance, the mesh size linearly grows up till a limited value as presented for both types of sections in Figure 6. Moreover, the picture shows an instantaneous velocity field on deformed finite element grid.

\section{Frequency tuning effects on the beam response}

The dynamic response of a slender beam under the natural wind is influenced by many circumstances. Parameters associated with the wind characteristics such as turbulence intensity, turbulent length or wind velocity principally follow from the character of a terrain where the structure is and they are ordinarily explicit. Much more influenceable features are related to the geometry of the examined beam and its modal characteristics. If the body is in aerodynamic shape, the flow regime around is rather potential than turbulent and the force action generated by the running air is reduced. Similarly, the wind load and the resulting response can be substantially decreased by the felicitous natural frequency tuning (given as the ratio between pitch to heave frequency) of the system as well as by proper selection of damping ratios.

Here, both sections are subjected to the numerical investigation on the classical flutter in dependence on the natural frequency tuning at constant damping characteristics. Two frequency ratios are considered, $\omega_{\alpha} / \omega_{h}=\langle 1.2,0.94\rangle$. The analysis consists in the incremental increase in the wind velocity until the flutter regime onset. The incremental size is $1 \mathrm{~m} / \mathrm{s}$, however, for higher accuracy a smaller rate can be employed. The results of the simulations are presented in Table 2. It includes values of the critical wind velocities determined by the flutter onset along with the main characteristics of the body oscillation in the post critical state such as the phase lag, $\phi$, and maximal attained amplitudes in both degrees of freedom, $A_{\alpha}$, $A_{h}$.Values in the brackets correspond to the tunnel experiment results published by present authors in $[13,14]$. Excepting one case in which an another type of instability was observed, the coupled mode regimes were achieved for both values of the frequency tuning; each at different wind speed and with different response characteristics.

Table 2. Characteristics of aeroelastic response for two values of frequency tuning.

\begin{tabular}{cccccc}
\hline & $\omega_{\alpha} / \omega_{h}$ & $u[\mathrm{~m} / \mathrm{s}]$ & $\phi\left[^{\circ}\right]$ & $\left.A_{\alpha}{ }^{\circ}\right]$ & $A_{h}[\mathrm{~mm}]$ \\
\hline & 0.94 & $8.0(14.9)$ & $32(20)$ & $9.0(6.0)$ & $4.0-5.0(18.0-26.0)$ \\
& 1.20 & $6.6(11.0)$ & $145(163)$ & $4.7(10.0)$ & $2.0-2.5(18.0)$ \\
& 0.94 & - & - & - & - \\
\hline & 1.20 & $15.0(14.0)$ & $55(163)$ & - & - \\
\hline
\end{tabular}

The streamlined section lost the stability due to the flutter only for ratio $\omega_{\alpha} / \omega_{h}=1.2$ at wind speed $u=15 \mathrm{~m} / \mathrm{s}$. The time-series of the torsional and vertical components are shown in Figure 7. Since the energy supplied to the system was very high, excessive oscillations arose and the numerical simulation based on Lagrangian-Eulerian description of motion collapsed before reaching steady-state vibrations. It was because of failure to meet an assumption of a low deformation of computation domains especially in heaving. For this reason the characteristics of this response are not relevant. When the ratio $\omega_{\alpha} / \omega_{h}=0.94$ was assumed, the simulation confirmed the experimental results and the section fell into the instability termed 
as the static divergence, see Figure 8. This is represented by the positive mean value of the rotational component accompanied by a buffeting response in the both generalized coordinates. Further velocity increasing led to the increase in the angle of rotation.

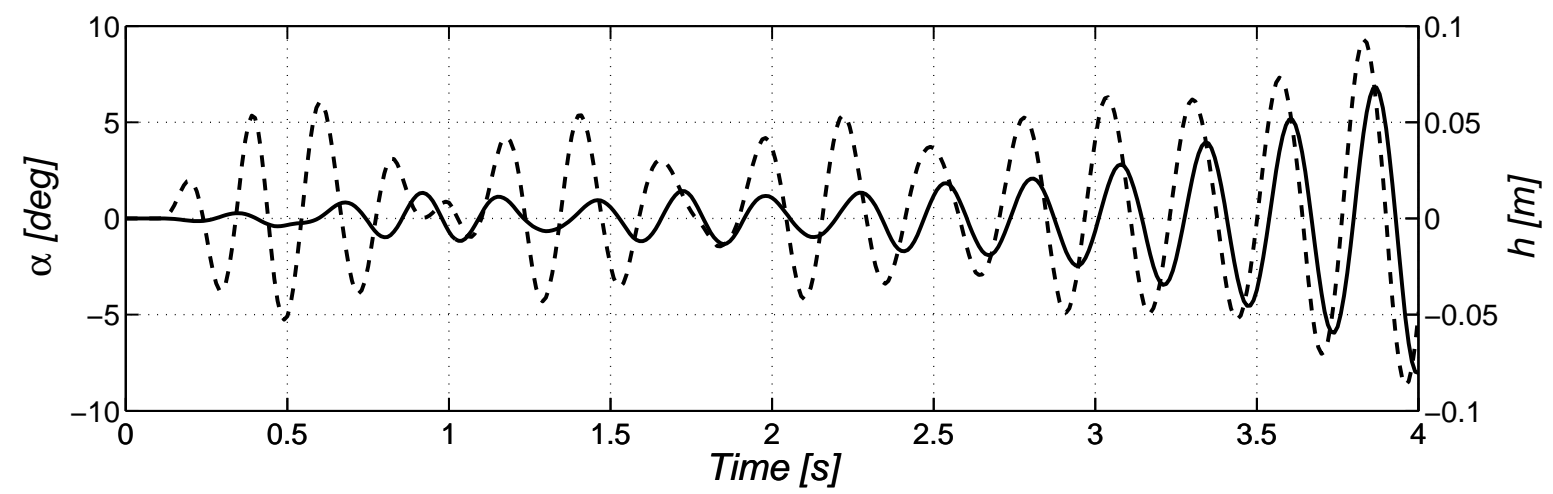

Figure 7. Streamlined section; Time-history record of heave (solid line) and rotation (dashed line) at flutter onset at $15 \mathrm{~m} / \mathrm{s} ; \omega_{\alpha} / \omega_{h}=1.2$.

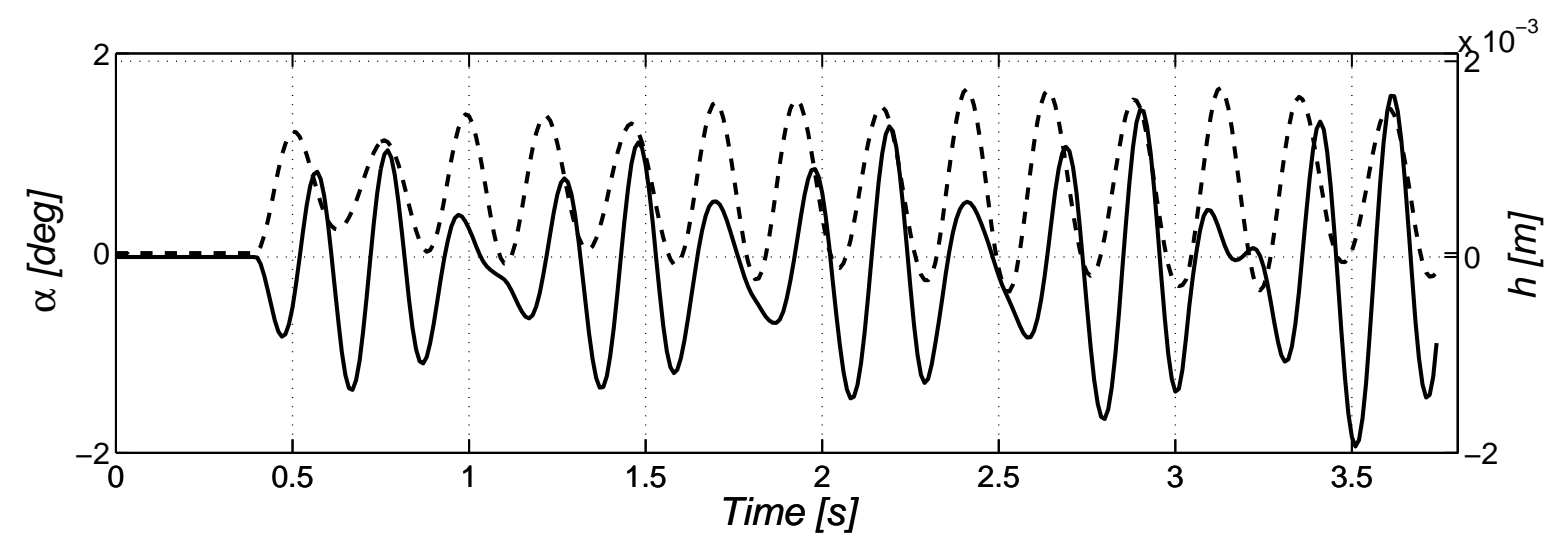

Figure 8. Streamlined section; Time-history record of heave (solid line) and rotation (dashed line) at flutter onset at $15 \mathrm{~m} / \mathrm{s} ; \omega_{\alpha} / \omega_{h}=0.94$.

Analogous numerical examination was used for the rectangular cross-section beam. This profile showed an unstable behaviour at much lower velocities. Because of the sharp angular edges on the windward side of the body, nonlinear aerodynamic alternating forces were generated that intensity is dependent proportionally on the angle of incidence of the wind.

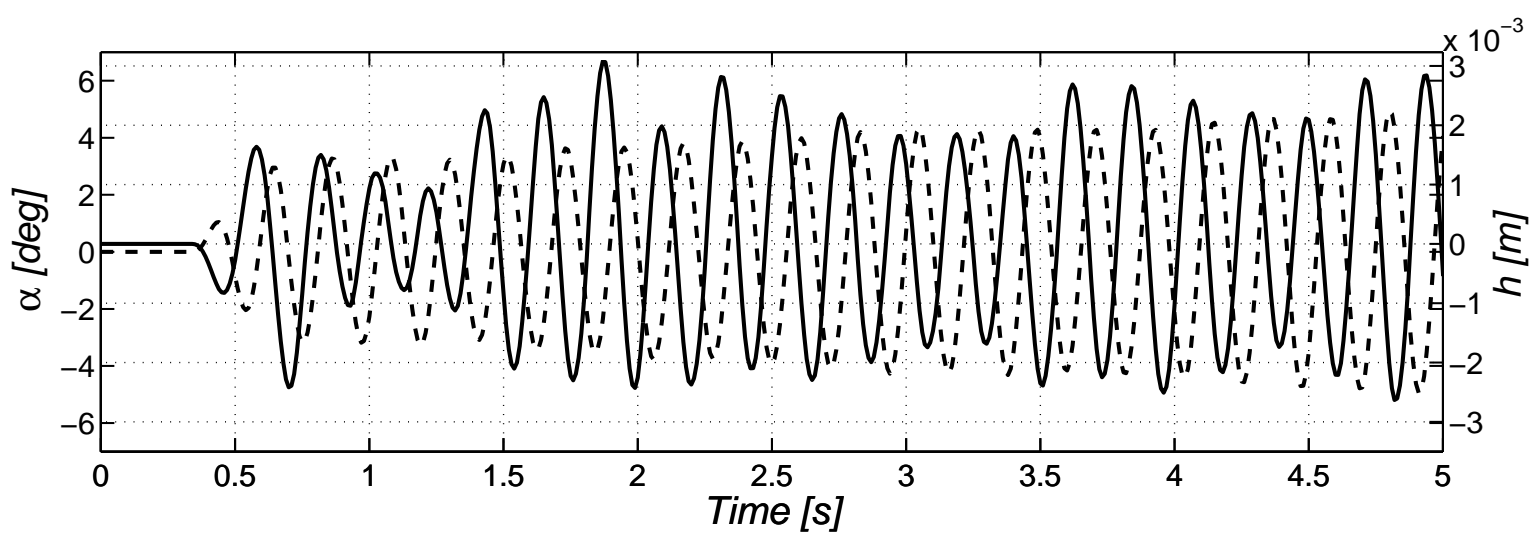

Figure 9. Rectangular section; Time-history record of heave (solid line) and rotation (dashed line) at flutter onset at $6 \mathrm{~m} / \mathrm{s} ; \omega_{\alpha} / \omega_{h}=1.20$. 
In Figure 9, the aeroelastic response is plotted at $u=6 \mathrm{~m} / \mathrm{s}$ and $f_{\alpha} / f_{h}=1.20$. From the picture is evident that rectangular exhibits a different oscillation behaviour compared to the streamlined section. The phase lag corresponds to the value of $\phi \approx 145^{\circ}$.

Provided that $f_{\alpha} / f_{h}=0.94$, the onset of the divergent oscillatory was considerably moved towards higher velocities. This tuning led to the relocation of the centre of the rotation reflected on a change in the phase lag, that reads $\phi \approx 32^{\circ}$. The response is demonstrated in Figure 10.

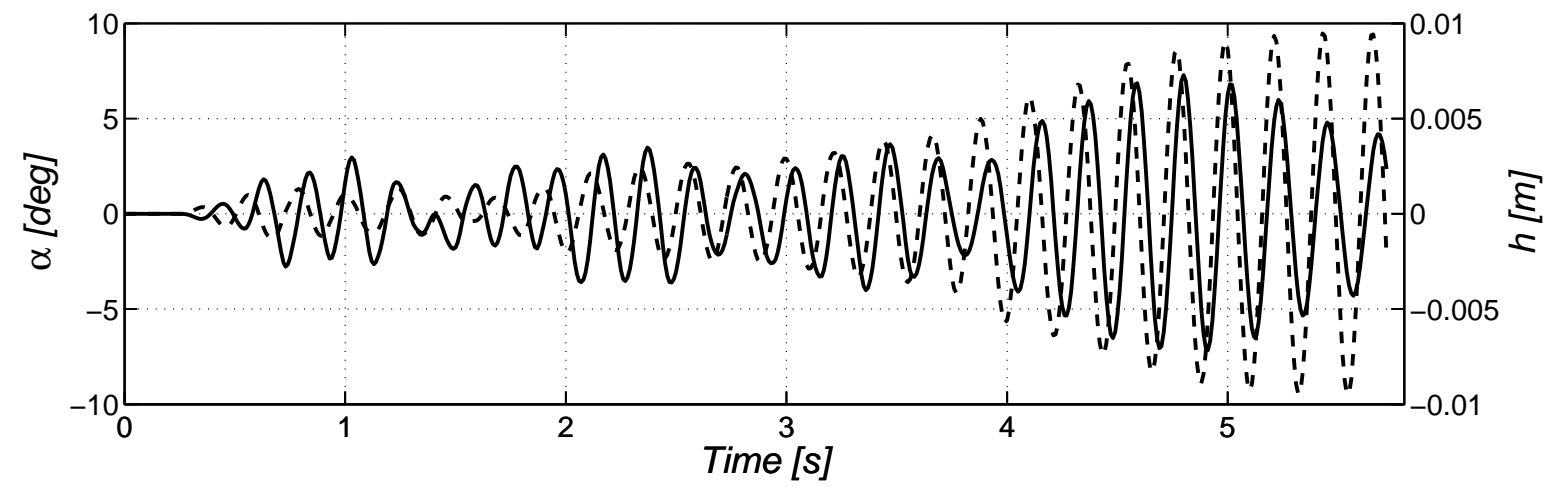

Figure 10. Rectangular section; Time-history record of heave (solid line) and rotation (dashed line) at flutter onset at $8 \mathrm{~m} / \mathrm{s} ; \omega_{\alpha} / \omega_{h}=0.94$.

\section{Determination of stability domains of the response}

The critical flutter condition occurs at a wind velocity at which the energy input is equal to the energy dissipated. Provided the structure in the windy conditions is displaced by an initial disturbance, its motion will either decay or diverge. Practically, such initial deflection can be caused by a random imperfection, e.g., gust of wind or other impulses. The aspects leading to stable or non-stable behaviour are not always transparent. The response character is influenced especially by the shape of the body and frequency tuning of the system, but other parameters like damping may also play a role.

Wind velocity range at which the energy gained from an air flow to the structure keeps the section oscillating, can be determined using a controlled wind speed method. To determine the response branches, the wind speed was increased continually up to the critical velocity. After reaching the critical state with the quasi steady-state oscillations, the wind speed was decreased again. The changes were regulated to maintain a slow rate $\Delta u=0.145 \mathrm{~m} / \mathrm{s}^{2}$. Using this technique, a "hysteresis" loop with two fundamental branches was observed. The lower branch is established once the wind velocity increases, while the upper branch appears during a decreasing wind speed regime. The critical state starts at the intersection of branches. This point represents a bifurcation corresponding to the lowest level of wind velocity at which the oscillating body is still able to extract energy from the flow and consequently remain in a single frequency coupled motion.

Under the procedure described above, the rectangular section have been analysed as depicted in Fig. 11. The velocity started at $5 \mathrm{~m} / \mathrm{s}$ and it grew at a steady rate $\Delta u$ till $7 \mathrm{~m} / \mathrm{s}$, corresponding to time $t=14 \mathrm{~s}$. Afterwards, the velocity was reduced at the same rate. During this process three relevant points were detected. At the first one, marked with green colour, 
onset regular oscillations in torsional mode were found at $u_{1}=5.7 \mathrm{~m} / \mathrm{s}$ in rising regime. After reaching $u_{2}=6.6 \mathrm{~m} / \mathrm{s}$, marked with red colour, the modes coupled and the both amplitudes escalated till velocity $7 \mathrm{~m} / \mathrm{s}$. The response maintained the pure coupled mode while velocity decreased till $u_{3}=5.7 \mathrm{~m} / \mathrm{s}$. From this point the rotation kept the smooth downward trend and an expressive regular amplitude fluctuation ensued in heaving vibration.
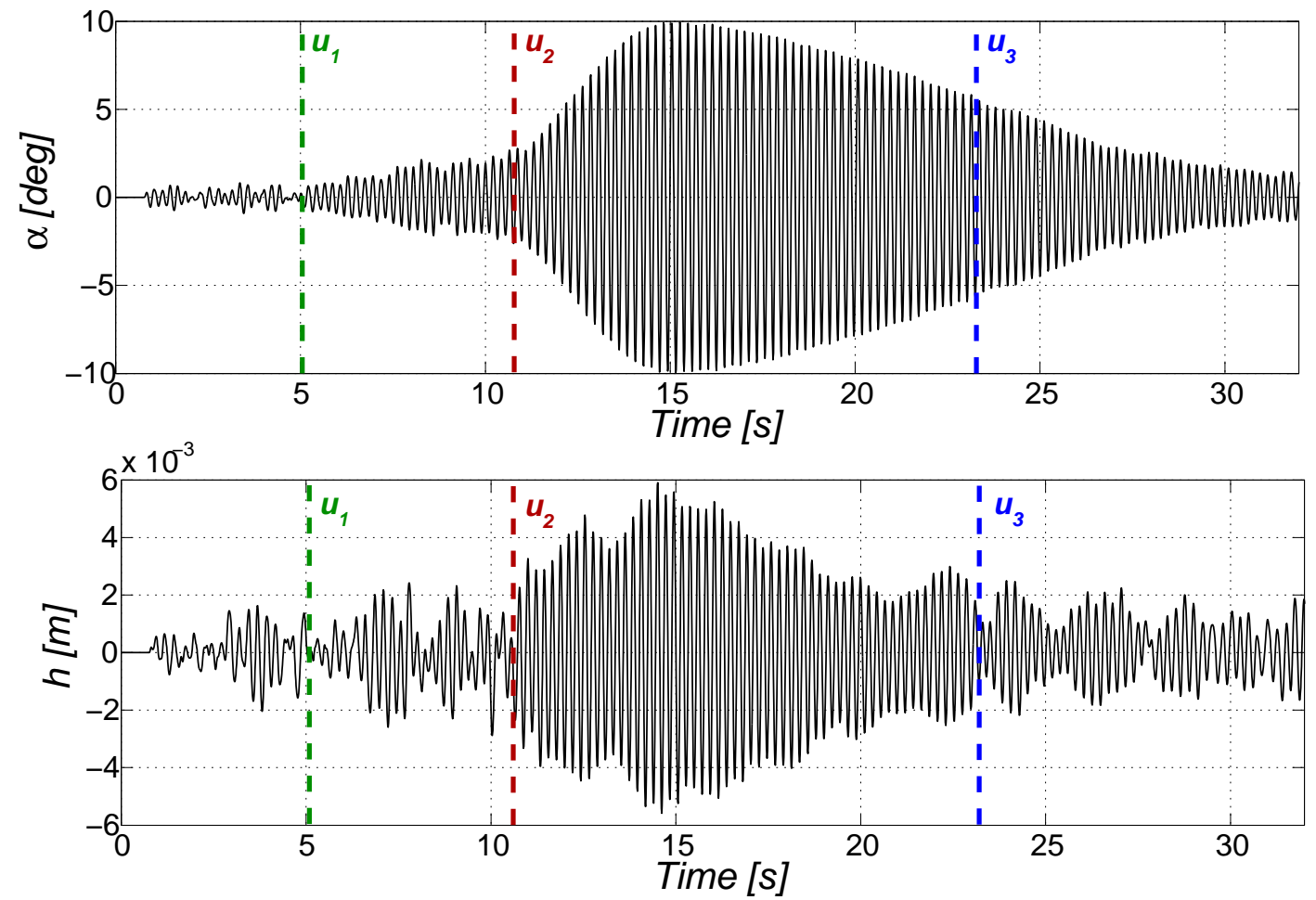

Figure 11. Rectangular section; Time-series of torsional (above) and heave (below) at variable velocity; $\omega_{\alpha} / \omega_{h}=1.2$.

This behaviour is also plotted on a velocity-amplitude plane in Figure 12. The hysteresis effect of the response is presented by loops $\mathrm{A}$ and $\mathrm{B}$ corresponding to the rotational and vertical motion, respectively, expressed in root-mean-square values. These graphs show that after reaching the critical velocity, the flutter motion continued even after the regulated decrease of the wind speed. The hysteresis loop delimits a critical velocity domain, where the flutter instability can occur under certain circumstances.

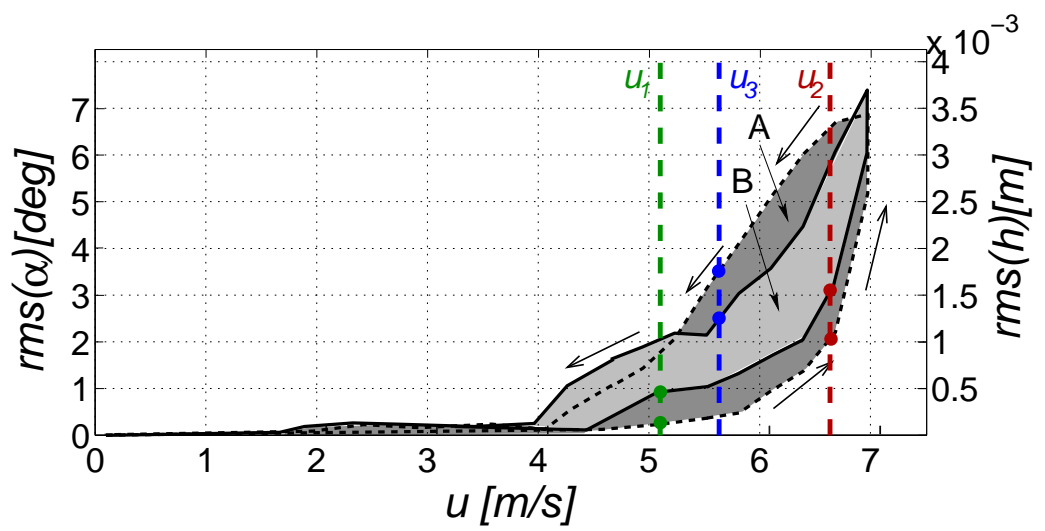

Figure 12. Heave (solid) and torsion (dashed) amplitude characteristics as functions of the velocity for rectangular section; $\omega_{\alpha} / \omega_{h}=1.20$. 
Strong hysteresis dependency was also observed in the wind tunnel experiments, but nevertheless with different response characteristics. In fact, during the experiment the velocity domains over the hysteresis loops were found wider and were located at higher magnitudes. Moreover, the lower branches of the loops for respective motion components were situated closer to the $u$-axis as the wind increased.

In Fig. 13, the stability diagrams of the coupled system with frequency ratio $f_{\alpha} / f_{h}=1.2$ are demonstrated for the rectangular section. Hysteresis loop and separation curves are displayed for both degrees of freedom. The separation curves divide the amplitude domain into two subdomains (A) and (B), respectively. Sub-domain (A) corresponds to initial disturbances resulting in the oscillation decaying towards the lower stable branch that shape is simplify into the straight line. If the initial deflection is located in the sub-domain (B), above the separation curve, an amplitude growth occurs towards the oscillation defined by the stable upper branch.

The separation occurs within the velocity interval $\left\langle\mathrm{u}_{\mathrm{B}}, \mathrm{u}_{\mathrm{C}}\right\rangle$. If the wind speed is within the range $\left\langle 0, \mathrm{u}_{\mathrm{B}}\right\rangle$, the coupled system is stable and an oscillation amplitudes decrease in time. When the velocity crosses over the limit $u_{B}$, the separation emerges correspondingly to the amplitude of the initial disturbance. It applies within the velocity interval $\left\langle\mathrm{u}_{\mathrm{B}}, \mathrm{u}_{\mathrm{C}}\right\rangle$. Above the critical velocity $\mathrm{u}_{\mathrm{C}}$, the profile looses stability due to self-induced aerodynamic forces. To establish a fundamental part of the separatrix in the interval $\left\langle\mathrm{u}_{\mathrm{B}}, \mathrm{u}_{\mathrm{C}}\right\rangle$, the initial disturbance in both degrees of freedom was applied simultaneously with the constant phase shift $\phi=180^{\circ}$ and the initial disturbance ratio $\alpha_{0} / h_{0}=22 \mathrm{rad} / \mathrm{m}$. It was applied for various wind velocities with a profile response measurement,in figure represented by symbol +. Afterwards, the points at the separation curve were identified by a simple observation of divergent motion.
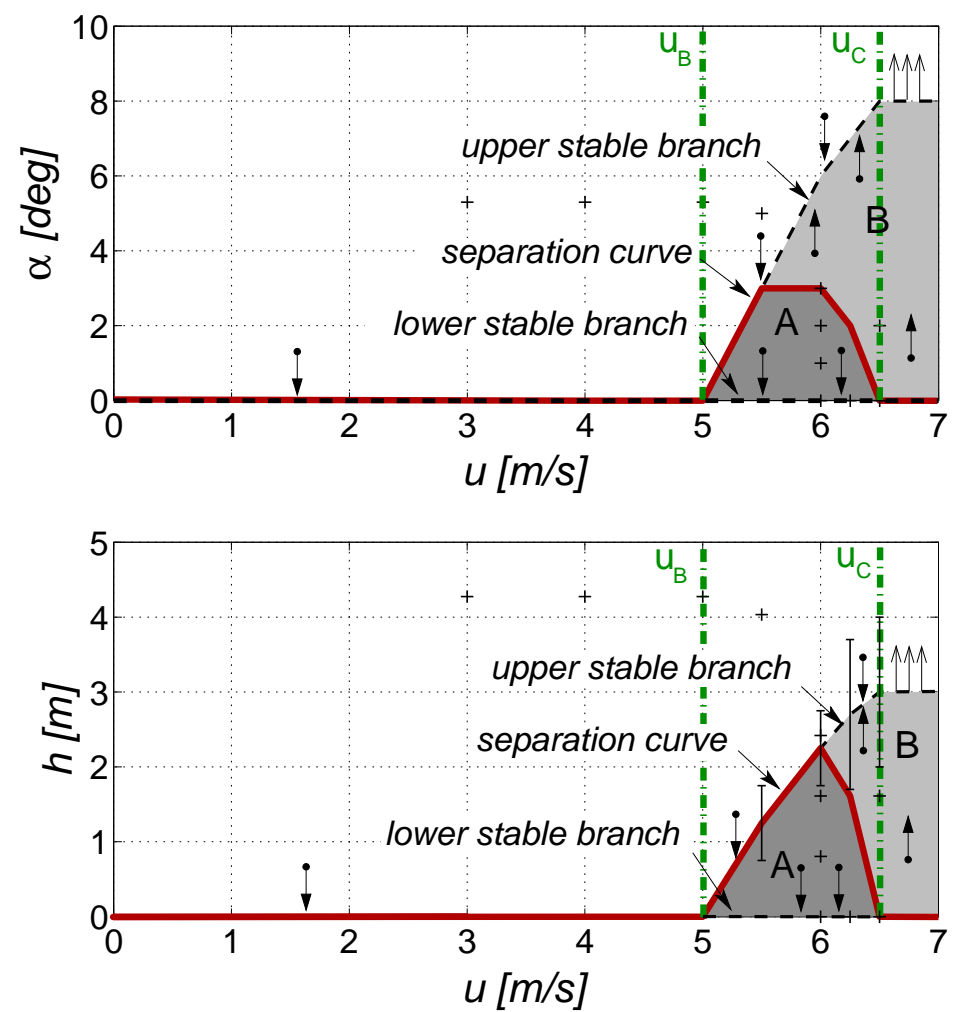

Figure 13. Separation curves of rectangular profile at the frequency ratio $\omega_{\alpha} / \omega_{h}=1.20$. 


\section{Conclusions}

This paper considered the character of the self-excited vibration of two cross-sections in the wind. Using the numerical model respecting fluid-structure interaction, a double-degreeof-freedom system were investigated with regard to the stability conditions before and within the oscillatory regime termed as the classical flutter. The basis of a theory of such a complex problem was introduced and formulated in detail. The modelling supposed the Newton's fluid, elasto-dynamic equations and arbitrary Eulerian-Lagrangian description of motion. In the analysis, the attention was paid to the elastic stiffness, damping and other DDOF system parameters influence on the type and shape of aero-elastic stability limits. It was focused primarily on the effect of the frequency tuning upon the stability onset.

We have shown that the instability onset can start in particular cases at lower wind speeds than those determined by the classical approaches when the influence of initial conditions and non-linear effects are neglected. Special attention was paid to the response of the section at onset of critical state and in reducing wind speed. The rectangular profile exhibited wide range of wind velocity for which the oscillations sustained the in coupled motion. This was demonstrated by a so-called hysteresis loop.

Using different initial body disturbances at different wind velocities, the separation curves were obtained. With the low-input disturbances, the conditions for flutter were met at lower wind velocities compared to the free vibration experiment. This fact follows from the relative position of the separation curve.

Besides the instability domains, an aero-elastic assessment has been carried out with respect to the various values of frequency ratios. Numerical examination shows that it is not necessary for flutter to occur if the heave frequency is lower that the torsion frequency, as is usually accepted. The profile in rectangular shape generating strong non-linear forces leads to flutter oscillations under the frequency ratio bellow one. It reflects on changes of section behaviour in the wind characterized by phase lag, oscillation frequency and critical velocity onset. All results following from this analysis were compared in a due manner with experimental research in the wind tunnel.

\section{Acknowledgments}

The support for this work was provided by the projects of Czech Grant Agency by project No. GAČR 103/09/0094, RVO: 68378297 and Grant Agency of the ASCR No. A200710902.

\section{REFERENCES}

[1] Hjorth-Hansen E., "Section model tests". Aerodynamics of Large Bridges, Balkema, Rotterdam, 1992.

[2] Irwin H.P.A.H., "Full aeroelastic model test. Aerodynamics of large Bridges, Balkema, Rotterdam, 1992.

[3] Frandsen J. B., "Numerical bridge deck studies using finite elements. Part I: flutter". Journal of Fluids and Structures 19, 171-191, 2004. 
[4] Koobus B., Farhart C., Tran H., "Computation of unsteady viscous flow around moving bodies using the $k-\varepsilon$ turbulence model on unstructured dynamic grids". Comput. Methods Appl. Mech. Eng. 190, 1441-1466, 2000.

[5] Shirai S., Ueda T., "Aerodynamic simulation by CFD on flat box girder of super-longspan suspension bridge". Journal of wind engineering and industrial aerodynamics 91 , 279-290, 2003.

[6] Shimada K., Ishihara T., "Application of modified $k-\varepsilon$ model to the prediction of aerodynamic characteristics of rectangular cross-section cylinders". J. Fluids Struct. 16 (4), 465-485, 2002.

[7] Bisplinghoff R.L., Ashley H., Halfman R.L., "Aeroelasticity”. Dover publications, Inc., Mineola, New York, 1996.

[8] Simiu E., Scanlan R.H., "Wind Effects on Structures". John Wiley \& Sons, New York, 1996.

[9] Donea J., Huerta A., "Finite Element Methods for Flow problems". John Wiley \& Sons Ltd, England, 2003.

[10] Tezduyar T.E., "Stabilised Finite Element Formulations for Incompressible Flow Computations". Advances in Applied Mechanics, volume 28, 1-44, 1992.

[11] Tezduyar T.E., Mittal S., Ray S.E., Shih R., "Incrompressible flow computations with stabilised bilinear and linear equal-order-interpolation velocity-pressure elements. Computer Methods in Applied Mechanics and Engineering”. 95, 221-242, 1992.

[12] Shakib F., Hughes T.J.R., "A new finite element formulation for computational fluid dynamics. IX. Fourier analysis of space-time Galerkin/least-squares algorithms", Comput. Methods Appl. Mech. Eng. 87(1), 35-58, 1991.

[13] Král R., Pospíšil S., Náprstek J., "Experimental analysis of frequency tuning influence on the response stability of bridge girders under wind action". In proceedings of ICWE13. Amsterdam: Dutch-Flemish Wind Engineering association, 2011, CD-ROM, 2011.

[14] Král R., Pospíšil S., Náprstek J., "Response of bluff and streamlined bridge girder in the wind as function of natural frequency tuning". In proceedings of 5th European and African conference on Wind engineering. Firenze: Firenze University Press, 2009, pp. 309-317, 2009. 